\title{
Utility of flouro-deoxy-glucose positron emission tomography/computed tomography in the diagnostic and staging evaluation of patients with primary CNS lymphoma
}

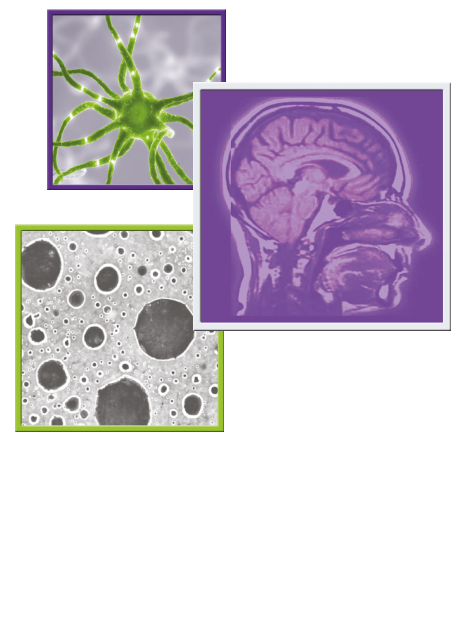

\author{
Meetakshi Gupta1, Tejpal Gupta*,1(iD), Nilendu Purandare², Venkatesh Rangarajan², Ameya \\ Puranik$^{2}$, Aliasgar Moiyadi ${ }^{3}$, Prakash Shetty ${ }^{3}$, Sridhar Epari ${ }^{4}$, Ayushi Sahay ${ }^{4}$ Abhishek \\ Mahajan $^{5}$, Amit Janu ${ }^{5}$, Bhausaheb Bagal ${ }^{6}$, Hari Menon $^{6}$, Sadhana Kannan, Rahul \\ Krishnatry ${ }^{1}$, Goda Jayant Sastri ${ }^{1}$ \& Rakesh Jalali ${ }^{1}$ \\ 1 Department of Radiation Oncology, TMH/ACTREC, Tata Memorial Centre, Homi Bhabha National Institute (HBNI), \\ Mumbai 400012, India \\ ${ }^{2}$ Department of Nuclear Medicine \& Molecular Imaging, TMH/ACTREC, Tata Memorial Centre, Homi Bhabha National Institute \\ (HBNI), Mumbai 400012, India \\ ${ }^{3}$ Department of Neuro-surgery, TMH/ACTREC, Tata Memorial Centre, Homi Bhabha National Institute (HBNI), \\ Mumbai 400012, India \\ ${ }^{4}$ Department of Pathology, TMH/ACTREC, Tata Memorial Centre, Homi Bhabha National Institute (HBNI), Mumbai 400012, India \\ ${ }^{5}$ Department of Radio-diagnosis, TMH/ACTREC, Tata Memorial Centre, Homi Bhabha National Institute (HBNI), \\ Mumbai 400012, India \\ ${ }^{6}$ Department of Medical Oncology, TMH/ACTREC, Tata Memorial Centre, Homi Bhabha National Institute (HBNI), \\ Mumbai 400012, India \\ ${ }^{7}$ Department of Clinical Research Secretariat, TMH/ACTREC, Tata Memorial Centre, Homi Bhabha National Institute (HBNI), \\ Mumbai 400012, India \\ *Author for correspondence: Tel.: +91 222740 5510; Fax: +91 222740 5061; tejpalgupta@rediffmail.com
}

\section{Practice points}

- MRI may not be able to reliably differentiate primary central nervous system (CNS) lymphoma (PCNSL) from morphologically similar lesions (glioblastoma, brain metastases and infection/inflammation).

- Flouro-deoxy-glucose positron emission tomography/computed tomography (18F-FDG-PET/CT) is a functional imaging modality that assesses glucose metabolism and has an established role in systemic lymphoma.

- Clinical utility of whole-body FDG-PET/CT in the diagnostic and staging evaluation of PCNSL has not been studied systematically.

- PCNSL has significantly higher FDG-uptake compared with other morphologically similar lesions in the brain.

- Whole-body FDG-PET/CT identifies occult systemic foci of lymphoma in a small proportion of patients presenting with disease apparently confined to the CNS.

Aim: Toprospectively assess the clinical utility of pretreatment flouro-deoxy-glucose positron emission tomography/computed tomography (18F-FDG-PET/CT) in patients with primary central nervous system (CNS) lymphoma (PCNSL). Materials \& methods: Patients with suspected/proven PCNSL underwent baseline whole-body 18F-FDG-PET/CT. Maximum standardized uptake value and tumor/normal tissue ratios were compared between CNS lymphoma and other histological diagnoses. Results: The mean maximum standardized uptake value ( 27.5 vs $18.2 ; \mathrm{p}=0.001)$ and mean tumor/normal tissue ratio ( 2.34 vs 1.53 ; $p<0.001)$ of CNS lymphoma was significantly higher than other histologic diagnoses. Five of $50(10 \%)$ patients with biopsy-proven CNS lymphomas had pathologically increased FDG-uptake at extraneuraxial sites uncovering systemic lymphoma. Conclusion: Pretreatment whole-body 18F-FDG-PET/CT provides valuable complementary information in the diagnostic and staging evaluation of patients with PCNSL to guide therapeutic decision-making.

Lay abstract: There is considerable overlap in conventional imaging features of primary central nervous system (CNS) lymphoma with other lesions such as glioblastoma and metastases. Flouro-deoxy- 
glucose positron emission tomography/computed tomography can reliably differentiate primary CNS lymphoma from brain lesions with similar morphology on conventional MRI. Based on significantly high flouro-deoxy-glucose-avidity of CNS lymphoma, semiquantitative flouro-deoxy-glucose positron emission tomography/computed tomography parameters were able to differentiate between brain lymphoma and nonlymphomatous lesions with acceptable diagnostic accuracy. A small proportion (10\%) of patients also had pathological flouro-deoxy-glucose-uptake in other body parts resulting in the diagnosis of systemic lymphoma with spread to the brain.

\section{Graphical abstract:}

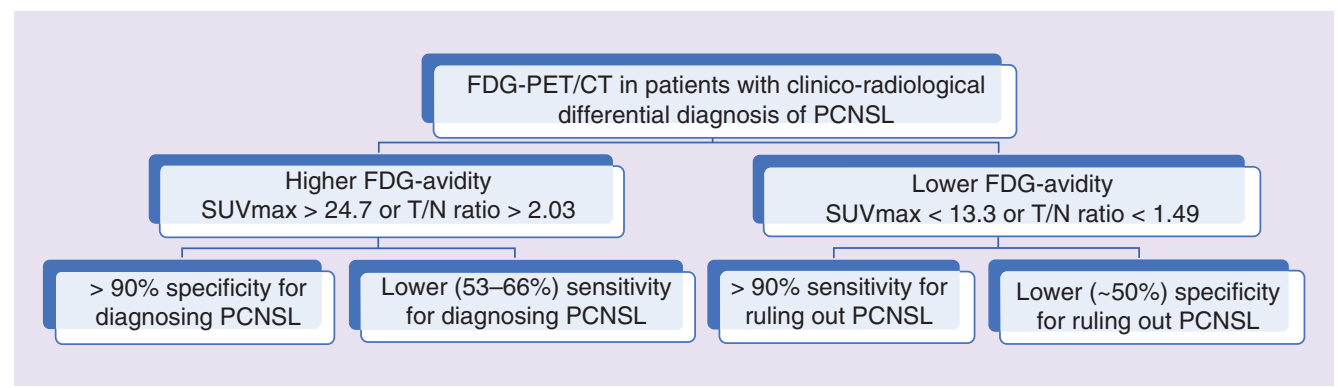

First draft submitted: 14 August 2019; Accepted for publication: 11 October 2019; Published online: 29 November 2019

Keywords: brain • FDG-PET/CT • lymphoma • MRI • systemic staging

Primary central nervous system (CNS) lymphoma (PCNSL) comprises $<2 \%$ of all primary brain tumors and $2-3 \%$ of all non-Hodgkin lymphoma [1,2]. Contrast-enhanced MRI is the recommended imaging modality for all suspected CNS tumors including PCNSL. In immunocompetent adults with primary CNS diffuse large B-cell lymphoma (DLBCL), MRI generally shows a solitary intracranial mass lesion (in nearly two-thirds of patients), mostly located in the cerebral hemispheres, basal ganglia, corpus callosum and deep periventricular regions with multiple lesions reported in approximately a third of patients [3]. Lesions of PCNSL are typically iso-intense on T1-weighted images, hypo-intense on T2-weighted images, that show homogenous and intense contrast enhancement, diffusion restriction and variable perifocal edema [3,4]. Since there is considerable overlap in the imaging characteristics of PCNSL with other common brain lesions such as glioblastoma, brain metastases and inflammatory or infectious pathologies, conventional morphologic MRI is generally insufficient to reliably and unequivocally differentiate between them. By definition, PCNSL is restricted to the brain, leptomeninges, spinal cord or eyes, without any evidence of involvement outside the neuraxis [1]. Any extraneuraxial involvement at initial diagnosis is considered as systemic lymphoma with secondary CNS involvement. Following the diagnosis of a CNS lymphoma, the extent of staging required to evaluate for a systemic lymphoma remains controversial [5,6]. Between $4-13 \%$ of patients presenting with disease apparently confined to the CNS have extraneuraxial involvement on systemic imaging [7]. The current recommendation of the International Primary CNS Lymphoma Collaborative Group is to perform a baseline CT scan of the chest, abdomen and pelvis along with testicular ultrasonography (in males) to detect any site(s) of systemic involvement in PCNSL [8]. The identification of systemic involvement in suspected PCNSL alters patient management significantly and adversely affects prognosis $[9,10]$.

Rapid advancements in imaging technology have now made it feasible to study various aspects of the tumor microenvironment such as hypoxia, angiogenesis, cellularity, proliferation and metabolism. Flouro-deoxy-glucose positron emission tomography/computed tomography (18F-FDG-PET/CT) that assesses glucose metabolism in the tumor remains by far the most widely used functional imaging modality in the diagnosis, staging, prognosis and monitoring of response in contemporary oncologic practice [11]. Over the years, the role of FDG-PET/CT in systemic lymphoma for staging at initial diagnosis, early interim response assessment after 1-2 cycles of chemotherapy and post-treatment surveillance following completion of therapy [12] has been firmly established. However, the clinical utility of baseline FDG-PET/CT in the diagnosis and staging of PCNSL has not been evaluated systematically. The primary aim of the study was to assess the diagnostic utility of baseline (pretreatment) brain FDG-PET/CT in patients with suspected PCNSL by reliably differentiating it from other lesions with similar morphology (i.e., glioblastoma, brain 
metastases, infectious/inflammatory lesions) on conventional neuroimaging. A secondary aim was to assess the utility of whole-body FDG-PET/CT for detection of systemic involvement in patients who present with disease apparently confined to the CNS.

\section{Materials \& methods}

\section{Study design \& participants}

The imaging study consisted of two parts; in Part A, adult patients with a strong clinicoradiological suspicion of PCNSL on conventional neuroimaging such as MRI (with or without spectroscopy and diffusion-weighted imaging) were included in the study and underwent baseline (pretreatment) whole-body FDG-PET/CT imaging after discussion in a multidisciplinary joint clinic. A planned neurosurgical intervention either limited to biopsy (stereotactic or open) or resection as deemed appropriate and safe by the operating neurosurgeon provided confirmatory histopathological diagnosis. Patients with deep seated lesions wherein biopsy was deemed hazardous, and in patients with obvious leptomeningeal enhancement, cerebrospinal fluid (CSF) was tested for malignant cell cytology and supplemented with flow-cytometry to arrive at a cytological diagnosis. Patients presenting with histopathologically proven CNS lymphoma (following brain or vitreous biopsy) were accrued directly in Part B and underwent whole-body FDG-PET/CT scan for baseline (pretreatment) staging before the initiation of definitive chemo(radio)therapy. The diagnostic utility of baseline FDG-PET/CT was measured using pathological diagnosis (biopsy/cytology) as reference standard. Any nonphysiologically increased FDG uptake outside the neuraxis suspicious of lymphomatous involvement was confirmed by either fine needle aspiration cytology or biopsy to establish the presence of systemic disease. Baseline (pretreatment) FDG-PET/CT was used for determining the incidence and patterns of systemic involvement in patients with histopathologically proven CNS lymphoma presenting with disease apparently confined to the neuraxis. Known cases of systemic lymphoma with secondary CNS involvement and patients with prior history of known malignancy with newly diagnosed brain lesion(s) were excluded from the study. The study was approved by the Institutional Ethics Committee and all patients provided written informed consent prior to study participation. All FDG-PET/CT scans were funded through a competitive study-specific intramural research grant, with the sponsor having no role in study design, conduct, analysis and reporting.

\section{Study procedures}

FDG-PET/CT imaging

After $6 \mathrm{~h}$ of fasting and confirming normal plasma glucose levels $(<150 \mathrm{mg} / \mathrm{dl}), 5 \mathrm{MBq} / \mathrm{kg}$ bodyweight of the radiotracer (18F-FDG) was injected intravenously. Static whole-body images (scan volume extending from skull vertex till mid-thigh) were obtained 45-60 min postinjection of the radioisotope on dedicated PET/CT scanners (Discovery ST, GE Healthcare, IL, USA and Astonish TF, Philips Healthcare, Guildford, UK). PET images were acquired on a time-of-flight system using approximately eight bed positions (60-90 s per bed position) and 50\% overlap for the emission study. PET images were reconstructed iteratively using the row action maximal likelihood algorithm. The corresponding CT with nonionic intravenous contrast was acquired at $120 \mathrm{kV}$ and set to auto $\mathrm{mA}$ as tube current for the CT. Attenuation correction of time-of-flight acquired data was done using postacquisition correction algorithms. Dead time correction and decay correction was also applied as appropriate. Two commonly used relative measures of FDG-PET/CT imaging, in other words, maximum standardized uptake value (SUVmax) and tumor/normal tissue $(\mathrm{T} / \mathrm{N})$ ratio were calculated using standardized and validated methodology. Region of interest (ROI) was placed on the tumor using image slices corresponding to maximum tracer uptake. SUVmax was expressed as a ratio of the maximum tissue radioactivity concentration 'c' of FDG in the selected ROI at time point ' $\mathrm{t}$ ' $(\mathrm{MBq} / \mathrm{kg})$ and decay corrected amount of injected $\mathrm{FDG}(\mathrm{MBq})$ divided by bodyweight $(\mathrm{kg})$. $\mathrm{T} / \mathrm{N}$ ratio also referred to as the uptake ratio was computed by dividing the SUVmax in an ROI by the SUVmax in a mirror image ROI placed manually in the contralateral hemisphere within normal uninvolved brain tissue. High-dose methotrexate-based chemotherapy with or without radiotherapy was offered to all fit patients with PCNSL as per the standard institutional protocol. Patients deemed unfit or ineligible for methotrexate were treated with alternative chemotherapy regimens or palliative radiotherapy based on physician discretion.

\section{Statistical considerations}

SUVmax and T/N ratios were calculated for all patients as described. Mean and median SUVmax and T/N ratio of patients with histopathologically proven CNS lymphoma was compared with the mean and median SUVmax and mean $\mathrm{T} / \mathrm{N}$ ratio of other histologic diagnoses (nonlymphomas) using the Mann-Whitney $\mathrm{U}$ test. Any p-value 


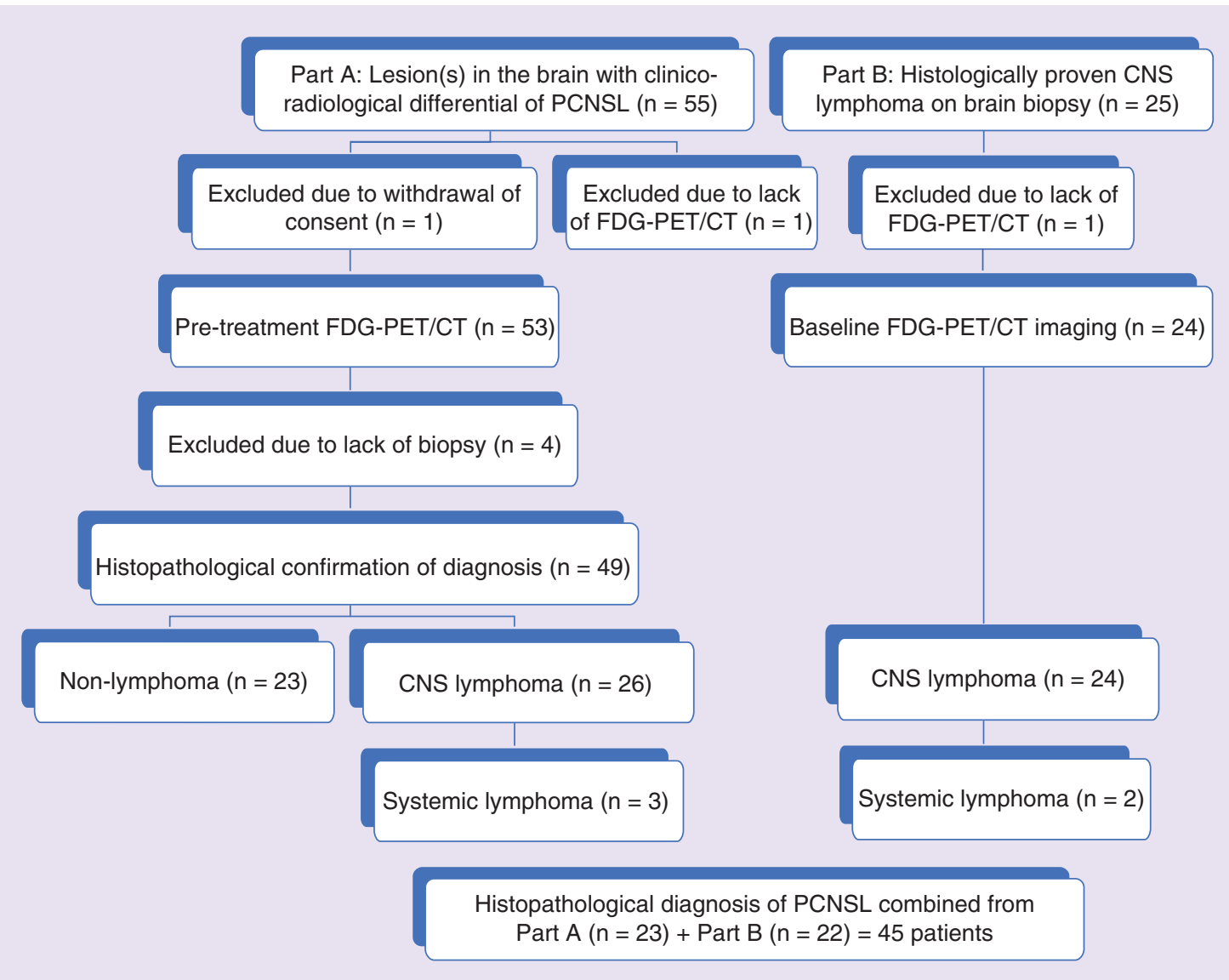

Figure 1. Schematic workflow of patients through the study.

CNS: Central nervous system; FDG-PET/CT: Flouro-deoxy-glucose positron emission tomography/computed tomography; PCNSL: Primary central nervous system lymphoma.

less than or equal to 0.05 was considered as statistically significant. Receiver operating characteristics (ROC) curves were generated to identify the optimal cut-offs of SUVmax and T/N ratios for reliably discriminating PCNSL from other histological diagnoses. Metrics of diagnostic accuracy such as sensitivity, specificity, negative predictive value (NPV) and positive predictive value (PPV) with corresponding 95\% confidence intervals (CI) were also calculated for the ROC defined cut-offs. Overall diagnostic accuracy was defined as the area under the curve of the ROC curves and was presented as a point estimate with corresponding 95\% CI. The incidence of systemic lymphoma (with 95\% CI) was calculated based on cytological or histological confirmation of extraneuraxial disease.

\section{Results}

Patient flow \& characteristics

Patient flow on the FDG-PET/CT imaging study conducted between 2014 and 2017 is described in Figure 1. A total of 55 patients consented and were accrued in Part A of the study as suspected PCNSL based on MRI after discussion in the multidisciplinary neuro-oncology joint clinic. Of them, $53(96 \%)$ patients underwent whole body FDG-PET/CT; however, diagnosis was confirmed histologically and/or cytologically in 49 (93\%) of 53 patients. Four of 53 (7\%) patients could not undergo histological/cytological confirmation of disease, one died waiting for biopsy while the other three were treated a clinicoradiological presumptive diagnosis of PCNSL. However, given the lack of histopathological diagnosis, they were excluded from the final analysis of diagnostic utility. All pretreatment FDG-PET/CT scans were reviewed independently and interpreted qualitatively by a senior imaging specialist, who reported the 'likely' diagnosis on FDG-PET/CT blinded to the final pathological diagnosis. Performance of baseline FDG-PET/CT using standardized metrics of diagnostic test accuracy with histology/cytology as reference standard has been reported in detail previously [13]. Twenty six of 49 (53\%) patients were proven to have DLBCL type of 
Table 1. Clinical characteristics of the study cohort $(n=80)$.

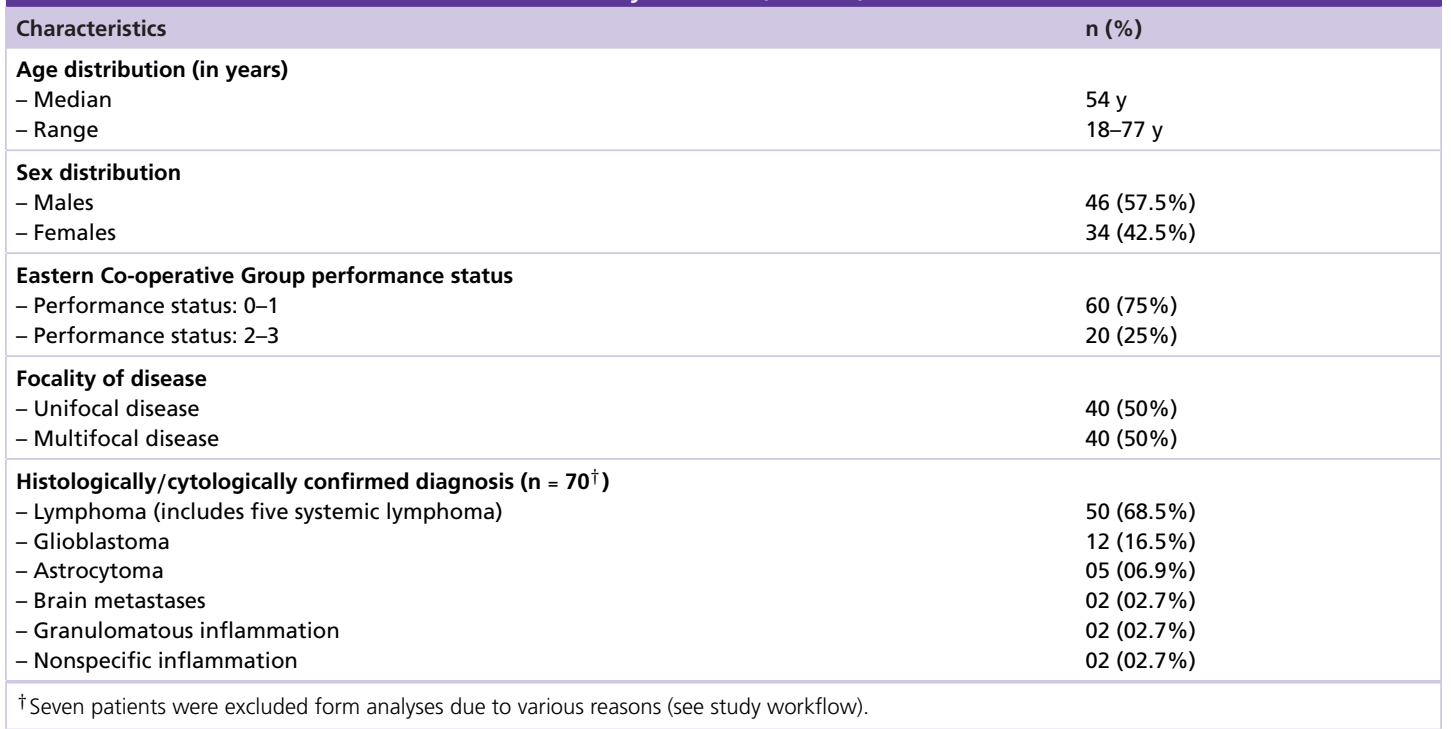

CNS lymphoma, including three patients with systemic involvement outside the neuraxis, which was subsequently confirmed pathologically. The remaining $23(47 \%)$ patients had histologic diagnoses other than lymphoma such as high-grade glioma $(n=17)$, infectious/inflammatory pathology $(n=4)$ and brain metastases $(n=2)$. All 26 patients with histological/cytological diagnosis DLBCL from Part A were also consented and included in Part B of the study. In addition, 25 patients referred after a histopathological diagnosis of DLBCL type of CNS lymphoma on brain/vitreous biopsy were consented directly on Part B of the study for undergoing pretreatment FDG-PET/CT. One (4\%) of these 25 patients worsened and died within 2 weeks of surgery prior to the baseline FDG-PET/CT and was excluded from analysis. Of the total of 50 patients (26 from Part A and 24 patients directly in Part B) with proven CNS lymphoma, 45 (90\%) patients were diagnosed on brain biopsy, four (8\%) on CSF cytology/flowcytometry and one (2\%) on vitreous biopsy. Two (8\%) of 24 patients in Part B had undergone complete resection of their solitary brain lesion that was confirmed as lymphoma on histopathology. Consequently, no FDG-avid lesion could be demonstrated on the postresection baseline FDG-PET/CT scan. In three (12\%) of 24 patients from Part B (biopsy-proven lymphoma), either the primary lesion in the brain could not be localized on the FDG-PET/CT $(n=2)$ or did not show any FDG-uptake $(n=1)$ precluding the calculation of semiquantitative FDG-PET/CT parameters. Two of them did not have identifiable brain lesion(s) on FDG-PET/CT; they were diagnosed as PCNSL on CSF flow-cytometry and vitreous aspirate respectively. The third patient had multiple, nonenhancing, signal changes in bilateral periventricular region which were iso-to-hyperintense on T2 and fluid attenuation inversion recovery images; however, differentially higher FDG-uptake compared with normal brain parenchyma was not demonstrated. Consequently, these five (10\%) patients had to be excluded from the total 50 patients, leaving 45 patients of CNS lymphoma for inclusion in the final analysis of diagnostic utility. However, these five were included in the analysis for systemic staging. Patient characteristics of the study cohort are briefly described in Table 1.

\section{Diagnostic accuracy}

There was significantly higher FDG-uptake in CNS lymphomas $(\mathrm{n}=45)$ compared with other histologic diagnoses $(\mathrm{n}=23)$ as illustrated in two representative cases from the study cohort (Figure 2). Box plots comparing SUVmax and $\mathrm{T} / \mathrm{N}$ ratio of CNS lymphomas versus nonlymphomas are depicted in Figure 3. The mean $( \pm$ standard deviation) SUVmax of CNS lymphomas was significantly higher at $27.5( \pm 12.53)$ compared with $18.2( \pm 9.75)$ for nonlymphomas $(\mathrm{p}=0.001)$. Similarly, the mean $\mathrm{T} / \mathrm{N}$ ratio of $\mathrm{CNS}$ lymphomas was significantly higher than other histologic diagnoses $(2.34 \pm 0.72$ vs $1.53 \pm 0.55 ; \mathrm{p}<0.001)$. ROC curves for defining optimal cut-offs of SUVmax and $\mathrm{T} / \mathrm{N}$ ratio for reliably differentiating CNS lymphomas from nonlymphomas are presented in Figure 4. The optimal cut-off of SUVmax for diagnosing CNS lymphoma was 18.8 with a sensitivity, specificity, PPV and NPV of 75\% (95\% CI: 53-81\%), 76\% (95\% CI: 52-90\%), 85\% (95\% CI: 74-92\%) and 52\% (95\% 

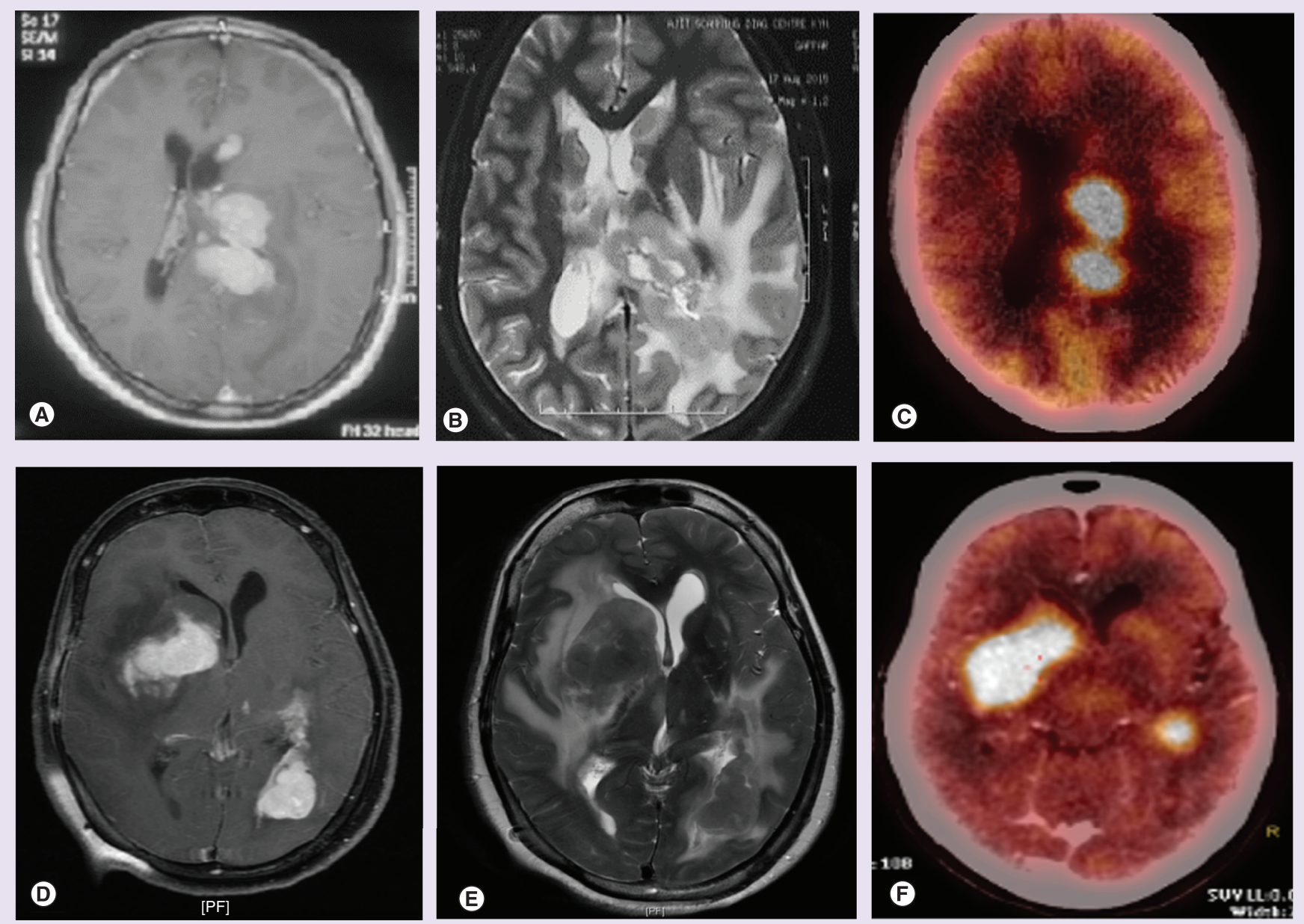

Figure 2. Representative case examples from the study cohort. Cranial MRI in the upper panel shows multiple, solid, periventricular lesions enhancing intensely on axial T1-weighted postcontrast image (A) and hypo-intense on axial T2-weighted image (B) with perifocal edema. Axial fused FDG-PET/CT image (C) shows significantly increased FDG-uptake with a maximum standardized uptake value (SUVmax) of 27 favoring lymphoma. Biopsy confirmed the diagnosis of PCNSL. MRI in the lower panel shows similar morphologic features on conventional neuroimaging in the form of multiple ill-defined lesions in the parietal lobe enhancing inhomogenously on axial T1-weighted postcontrast image (D) and iso-intense on axial T2-weighted image (E) with perifocal edema. In contrast to PCNSL, axial fused FDG-PET/CT image (F) shows only moderately increased FDG-uptake in the lesions with SUVmax of 12 suggestive of nonlymphomatous pathology. Diagnosis was confirmed as glioblastoma on surgical decompression.

FDG: Flouro-deoxy-glucose; FDG-PET/CT: Flouro-deoxy-glucose positron emission tomography/computed tomography; MRI: Magnetic resonance imaging; PCNSL: Primary CNS lymphoma.

CI: 42-63\%), respectively. Similarly, the optimal cut-off of $\mathrm{T} / \mathrm{N}$ ratio was 1.66 with a sensitivity, specificity, PPV and NPV of 84\% (95\% CI: 68-92\%), 81\% (95\% CI: 60-95\%), 90\% (95\% CI: 79-96\%) and 69\% (95\% CI: 54-81\%), respectively. The sensitivity of FDG-PET/CT in diagnosing a CNS lymphoma increased to $>90 \%$ at an SUVmax cut-off of 13.3 and $\mathrm{T} / \mathrm{N}$ ratio cut-off of 1.49; however, this was at the cost of reduced specificity (just above $50 \%$ for both). Conversely, a SUVmax cut-off value of 24.7 and a $\mathrm{T} / \mathrm{N}$ ratio cut-off value of 2.03 substantially increased the specificity of FDG-PET/CT (>90\%) for the diagnosis of CNS lymphoma, albeit at the cost of lower sensitivity (53 and 66\% for SUVmax and T/N ratio, respectively). Area under the curve of ROC-defined cut-off values of SUVmax (18.8) and T/N ratio (1.66) provided an overall accuracy of $75 \%(95 \%$ CI: $65-90 \%)$ and $83 \%$ (95\% CI: 72-94\%), respectively. 

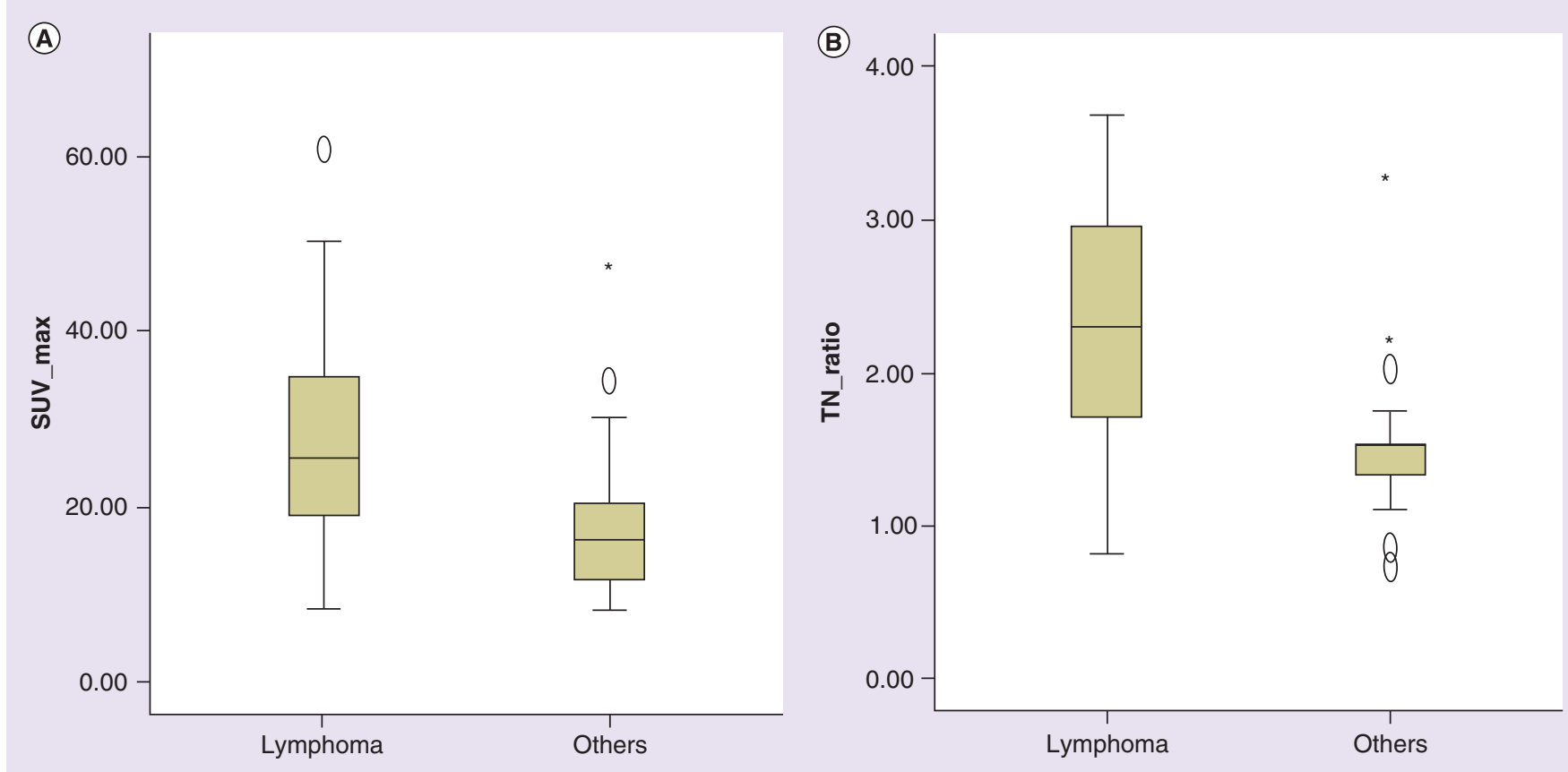

Figure 3. Box-plots of quantitative flouro-deoxy-glucose positron emission tomography/computed tomography parameters. Boxplots comparing SUVmax (A) and T/N ratio (B) between central nervous system lymphoma and nonlymphomatous histology. Note significantly higher SUVmax and $\mathrm{T} / \mathrm{N}$ ratio in lymphoma.

SUVmax: Maximum standardized uptake value; T/N: Tumor/normal tissue.

\section{Systemic staging}

Five of the 50 patients with proven DLBCL type of CNS lymphomas had nonphysiologically increased FDG-avidity at extraneuraxial sites, which were subsequently confirmed as sites of involvement by lymphoma on fine needle aspiration cytology/biopsy. The sites of extraneuraxial uptake in these five patients included bone, abdominal nodes, paratesticular mass, adrenal glands and soft tissue deposits in muscles and subcutaneous tissues. The overall incidence of detecting systemic lymphoma in patients who presented with disease apparently confined to the CNS was $10 \%$ (95\% CI: $3.3-21.8 \%)$.

\section{Discussion}

This study assessed the clinical utility of baseline whole-body FDG-PET/CT in the diagnosis and staging of patients with suspected and/or proven PCNSL. It clearly demonstrates significantly higher FDG-uptake in CNS lymphomas compared with its common mimics on conventional neuroimaging (glioblastoma, metastases and inflammatory/infectious lesions). The specificity of FDG-PET/CT increases significantly ( $>90 \%)$ with increasing SUVmax and $\mathrm{T} / \mathrm{N}$ ratio, although at the cost of decreased sensitivity. Thus, any lesion in the brain with a SUVmax of $>25$ and $\mathrm{T} / \mathrm{N}$ ratio $>2$ is highly likely to be a CNS lymphoma. Conversely, the sensitivity of SUVmax and $\mathrm{T} / \mathrm{N}$ ratio of 13.3 and 1.49 respectively is $>90 \%$, suggesting that any lesion with lesser values is highly unlikely to be lymphomatous in origin. Although histopathology remains the gold-standard for the diagnosis of PCNSL, whole-body FDG-PET/CT provides valuable complementary information that can influence decision-making, particularly in the setting of deep-seated lesions not amenable to a safe biopsy. Yamaguchi et al. [14] proposed the use of very high FDG-uptake combined with substantial regression in tumor size after one week of corticosteroid administration for diagnosing PCNSL in deep-seated brain lesions without histologic confirmation.

The results of this study are generally in accordance with previously published literature on FDG-avidity in PCNSL. Researchers from Duke University (NC, USA) [15] were the first to report increased FDG-uptake in a series of ten patients with PCNSL, which was significantly higher than lower grade gliomas, but not different from anaplastic astrocytoma. Unfortunately, this was largely based on visual and qualitative interpretation without any quantification. Selected studies that include greater than five patients reporting semiquantitative FDG-PET/CT 


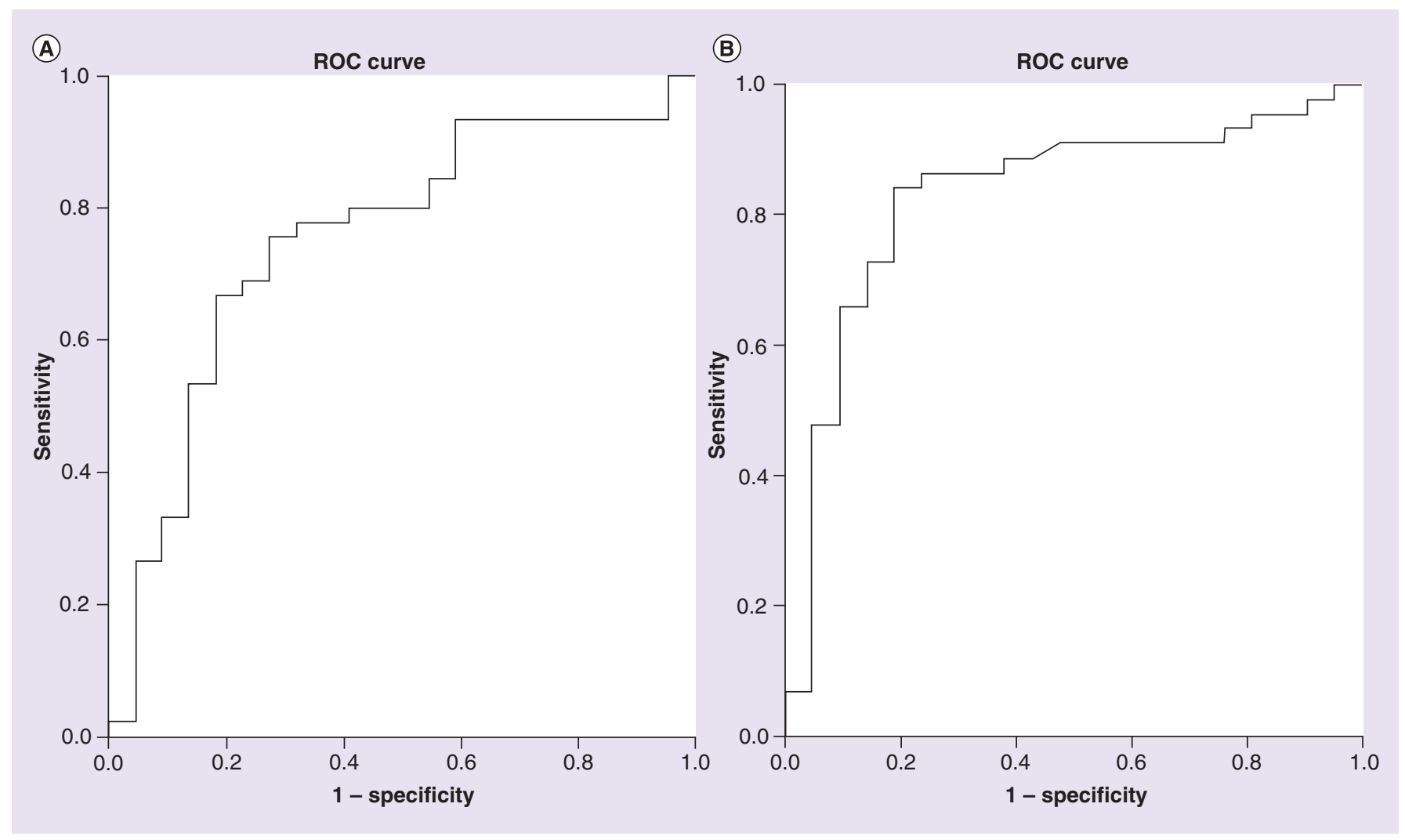

Figure 4. Receiver operating characteristics curves of quantitative flouro-deoxy-glucose positron emission tomography/computed tomography parameters. ROC curves of SUVmax (A) and T/N ratio (B) in the diagnosis of central nervous system lymphoma. Note that the upper left corner of the respective curves provides the most optimal cut-off of SUVmax and T/N ratio for diagnosing lymphoma. ROC: Receiver operating characteristic; SUVmax: Maximum standardized uptake value; T/N: Tumor/normal tissue.

parameters in PCNSL are summarized chronologically in Table 2 [16-31]. The reported mean/median values, however, have been variable, possibly due to differences in technical performance (image acquisition and quantitation), small number of patients in individual studies and interobserver variability. Most studies reported good utility of FDG-PET/CT in differentiating PCNSL from high-grade gliomas and brain metastases with some even providing ROC-defined cut-offs for the semiquantitative PET parameters with clinically acceptable diagnostic accuracy. However, other studies reported substantial overlap of SUVmax and T/N ratios between PCNSL and other nonlymphomatous CNS lesions, thereby questioning the incremental value of added FDG-PET/CT in the diagnostic evaluation of suspected PCNSL. In a systematic review and meta-analysis of eight retrospective studies involving a total of 129 patients, Zou et al. [32] reported good diagnostic performance of FDG-PET and FDG-PET/CT for diagnosing PCNSL with an overall pooled sensitivity of 0.88 (95\% CI: $0.80-0.94)$, specificity of 0.86 (95\% CI: 0.73-0.94), positive likelihood ratio of 3.99 (95\% CI: 2.31-6.90), negative likelihood ratio of 0.11 (95\% CI: 0.04-0.32) and diagnostic odds ratio of 33.40 (95\% CI: 10.40-107.3). Area under the summary ROC curve and Q-index (point on summary ROC curve where sensitivity equals specificity), measures of comprehensive diagnostic effect were acceptably high at 0.9192 and 0.8525 , respectively. Apart from the anatomic and functional imaging, modern technological advances including proteochemical, immunephenotypic and molecular genetic analyses of CSF can also provide high diagnostic accuracy in PCNSL [33].

The need for systemic staging in patients with biopsy-proven brain lymphoma has always been debated [5-7]. The current International Primary CNS Lymphoma Collaborative Group guidelines [8] recommend baseline CT scan of the thorax, abdomen and pelvis along with testicular ultrasonography (in males) for systemic staging keeping whole-body FDG-PET/CT as an optional investigation. However, it is being increasingly appreciated and acknowledged that, apart from its diagnostic utility, whole-body FDG-PET/CT can easily identify site(s) of extraneuraxial uptake indicating presence of systemic lymphoma with CNS involvement rather than PCNSL. In a large retrospective study [34] involving 262 patients with newly diagnosed lymphomatous brain lesions, 24 (11.8\%) 


\begin{tabular}{|c|c|c|c|c|}
\hline Author [Ref.] & Number of PCNSL patients & Mean ( \pm SD) of SUVmax & Mean $( \pm S D)$ of $T / N$ ratio & [Ref.] \\
\hline Kosaka et al. & 07 & $22.1( \pm 5.0)$ & $2.31( \pm 0.7)$ & [17] \\
\hline Kawai et al. & 17 & $13.5( \pm 5.4)$ & 2.54 (SD not reported) & [18] \\
\hline Kawase et al. & 13 & $13.9( \pm 5.7)$ & $2.74( \pm 1.25)$ & [19] \\
\hline Das et al. & 20 & $15.7( \pm 0)$ & Not reported & [21] \\
\hline Ghesquieres et al. & 24 & $13.9( \pm 9.3)$ & Not reported & [22] \\
\hline Okada et al. & 07 & $20.4( \pm 8.9)$ & Not reported & [23] \\
\hline Yamaguchi et al. & 19 & $22.6( \pm 12.6)$ & $2.79( \pm 0.91)$ & [24] \\
\hline Mercadal et al. & 14 & 17.0 (range 6-39) & Not reported & [25] \\
\hline Albano et al. & 38 & $15.6( \pm 9.2)$ & $3.1( \pm 1.7)$ & [30] \\
\hline Zhou et al. & 40 & $17.7( \pm 7.4)$ & $2.46( \pm 1.04)$ & [31] \\
\hline Present study & 45 & $27.5( \pm 12.53)$ & $2.34( \pm 0.72)$ & \\
\hline
\end{tabular}

$\dagger$ Median (not mean) value with range in parentheses.

PCNSL: Primary CNS lymphoma; SD: Standard deviation; SUVmax: Maximum standardized uptake value; T/N ratio: Tumor/normal tissue ratio.

patients were detected to have non-CNS malignancies (19 with systemic lymphoma and five with other secondary malignancies) on systemic staging, highlighting the importance of such evaluation. On the contrary, Suh et al. [35] recently reported very low diagnostic yield (2\%, six of 304 patients, 95\% CI: $0.7-4.3 \%)$ of whole-body FDGPET/CT for initial systemic staging in PCNSL. Somewhat more disturbing was double the number of false-positive findings (4\%, 13 of 304 patients, 95\% CI: 2.3-7.2\%) leading to further unnecessary procedures, re-igniting the debate on the extent of systemic staging. The $10 \%$ incidence of systemic lymphoma observed in this study in patients who present with disease apparently confined to the CNS is in accordance with previously published data $[7,34]$. Though seemingly uncommon, this has major diagnostic, prognostic and therapeutic implications $[9,10]$ that supports the current recommendation of pretreatment systemic staging in patients with PCNSL. Based on the results of this prospective study as well as previously published literature, baseline whole-body FDG-PET/CT should now be strongly considered as a useful imaging modality (adjunctive to cranial MRI) in the diagnostic and staging evaluation patients with PCNSL.

\section{Strengths \& limitations}

The strength of the study lies in its prospective design that included consecutive patients with clinicoradiological suspicion of PCNSL on conventional imaging such as MRI (with or without spectroscopy and diffusion-weighted imaging) who underwent pretreatment FDG-PET/CT imaging prior to definitive diagnosis. Such a design considerably reduces the bias inherent to any retrospective analysis. Quantification and calculation of FDG-PET/CT parameters was done using standardized validated methodology by a single observer eliminating potential interobserver bias. Though some patients were treated on a presumptive diagnosis of PCNSL, only patients with histologically/cytologically confirmed disease were included in the final analysis. All CNS lymphoma patients with increased FDG-uptake at extraneuraxial sites also underwent cytological/pathological confirmation to establish the diagnosis of systemic lymphoma. This is the largest study to date that compares quantitative FDG-PET/CT parameters between CNS lymphoma and other brain lesions with similar radiographic appearance. Despite these strengths, some caveats and limitations remain. A significant proportion of patients had undergone baseline MRI scans at outside centers with possibly different acquisition protocols for various sequences and variable magnet strengths that could potentially interfere with interpretation. Perfusion imaging and diffusion-weighted imaging including apparent diffusion coefficient maps were not routinely available on the baseline MRI scans in all patients. It has recently been demonstrated that perfusion and/or diffusion-weighted MRI can differentiate glioblastoma 
from PCNSL with high diagnostic accuracy [36-38]. In addition, this study did not use modern radiomic approaches such as support vector machine classifiers [39] based on textural features of postcontrast T1-weighted images that can more reliably differentiate glioblastoma from lymphoma compared with semantic MRI features. It is common knowledge that SUVs can be affected by several factors, including technical performance of the scan [40]. Nearly $10 \%$ of patients underwent FDG-PET/CT at outside centers with possible differences in quantitation of imaging parameters compared with the institutional protocol. At last, patients with no significant increase in FDG-avidity in the brain lesions were excluded from the quantitative analysis, potentially introducing bias.

\section{Conclusion}

MRI remains the recommended standard first-line imaging modality in the diagnostic evaluation of patients with intracranial lesions including PCNSL. Although histology remains the gold-standard for diagnosis, whole-body FDG-PET/CT provides valuable complementary information in the evaluation of patients with suspected PCNSL that can be used for presumptive diagnosis to guide therapeutic decision-making, particularly in deep-seated lesions not amenable to safe biopsy coupled with negative CSF cytology and flow-cytometry. In addition, it also identifies clinically occult foci of systemic lymphoma in a small proportion of patients with resultant implications for diagnosis, prognosis and therapy.

\section{Author contributions}

M Gupta and T Gupta are co-first authors contributing equally to the work. T Gupta is the guarantor of integrity of the entire study. The study concepts and design were given by T Gupta. M Gupta, A Janu, A Mahajan and T Gupta contributed in literature research. The clinical studies were done by N Purandare, V Rangarajan, A Puranik, B Bagal and H Menon. The experimental studies/data analysis was done by P Shetty, A Moiyadi, A Sahay and S Epari. Statistical analysis was done by M Gupta, S Kannan and T Gupta. M Gupta and T Gupta were involved in manuscript preparation. Manuscript editing was done by R Krishnatry, GJ Sastri and R Jalali.

\section{Acknowledgments}

This research article was presented in part at the 5th Quadrennial Meeting of the World Federation of Neuro-Oncologic Societies (WFNOS) at Zurich (Switzerland) in May 2017.

\section{Financial \& competing interests disclosure}

This work was partly supported by a competitive intramural research grant from Tata Memorial Centre. All FDG-PET/CT scans on the study were funded through a competitive intramural research grant (TMC IEC Project No. 145) secured by the Principal Investigator-cum-Corresponding Author. However, the sponsor had no role in the study design, conduct, data collection, analysis or reporting of results. The authors have no other relevant affiliations or financial involvement with any organization or entity with a financial interest in or financial conflict with the subject matter or materials discussed in the manuscript apart from those disclosed.

No writing assistance was utilized in the production of this manuscript.

Ethical conduct of research

All procedures performed in the study (Project No. 145) were in accordance with the ethical standards of the Institutional Ethics Committee (IEC) of Tata Memorial Centre, India that functions in accordance with the 1964 Helsinki declaration and its later amendments or comparable ethical standards. All the patients provided written informed consent before participation in the study.

Open access

This work is licensed under the Attribution-NonCommercial-NoDerivatives 4.0 Unported License. To view a copy of this license, visit http://creativecommons.org/licenses/by-nc-nd/4.0/

\section{References}

Papers of special note have been highlighted as: $\bullet$ of interest; $\bullet \bullet$ of considerable interest

1. Rubenstein J, Ferreri AJ, Pittaluga S. Primary lymphoma of the central nervous system: epidemiology, pathology and current approaches to diagnosis, prognosis and treatment. Leuk. Lymphoma 49(Suppl. 1), 43-51 (2008).

2. Han CH, Batchelor TT. Diagnosis and management of primary central nervous system lymphoma. Cancer 123(22), 4314-4324 (2017). 
3. Kuker W, Nagele T, Korfel A et al. Primary central nervous system lymphomas: MRI features at presentation in 100 patients. J. Neurooncol. 72(2), 169-177 (2005).

- Describes MRI features of primary central nervous system (CNS) lymphoma.

4. Tang YZ, Booth TC, Bhogal P, Malhotra A, Wilhelm T. Imaging of primary central nervous sytem lymphoma. Clin. Radiol. 66(8), 768-777 (2011).

5. O’Neill BP, Dinapoli RP, Kurtin PJ, Habermann TM. Occult systemic non-Hodgkin's lymphoma (NHL) in patients initially diagnosed as primary central nervous system lymphoma (PCNSL): how much staging is enough? J. Neurooncol. 25(1), 67-71 (1995).

6. Mohile NA, DeAngelis LM, Abrey LE. Utility of body FDG-PET in staging primary central nervous system lymphoma. Neuro Oncol. 10(2), 223-228 (2008).

7. Scott BJ, Douglas VC, Tihan T, Rubenstein JL, Josephson SA. A systematic approach to the diagnosis of suspected central nervous system lymphoma. JAMA Neurol. 70(3), 311-319 (2013).

- Provides a useful and practical algorithm for diagnosis in patients who present with intracranial lesion(s) suspicious for primary CNS lymphoma (PCNSL).

8. Abrey LE, Batchelor TT, Ferreri AJ et al. Report of an international workshop to standardize baseline evaluation and response criteria for primary CNS lymphoma. J. Clin. Oncol. 23(22), 5034-5043 (2005).

-• The International PCNSL Collaborative Group recommends whole-body computed tomography and bone-marrow biopsy in patients with PCNSL as part of baseline diagnostic work-up highlighting the need for systemic staging in PCNSL.

9. Malecek M, Petrich AM, Rozell S et al. Frequency, risk factors, and outcomes of central nervous sytem relapse in lymphoma patients treated with dose-adjusted EPOCH plus rituximab. Am. J. Hematol. 92(11), 1156-1162 (2017).

10. El-Galaly TC, Cheah CY, Bendtsen MD et al. Treatment strategies, outcomes, and prognostic factors in 291 patients with secondary CNS involvement by diffuse large B-cell lymphoma. Eur. J. Cancer 93, 57-68 (2018).

11. Wood KA, Hoskin PJ, Saunders MI. Positron emission tomography in onclogy: a review. Clin. Oncol. 19(4), 237-255 (2007).

12. Cronin CG, Swords R, Truong MT et al. Clinical utility of PET/CT in lymphoma. AJR Am. J. Roentgenol. 194(1), W91-W103 (2010).

- Summarizes the established role of flouro-deoxy-glucose positron emission tomography/computed tomography in systemic lymphoma.

13. Gupta T, Gupta M, Purandare N et al. Diagnostic performance of baseline 18F-flouro-deoxy-glucose positron emission tomography/computed tomography in patients with clinico-radiological suspicion of primary central nervous system lymphoma. Ann. Hematol. Oncol. 6(1), 1228 (2019).

14. Yamaguchi S, Hirata K, Kaneko S et al. Combined use of 18F-FDG PET and corticisteroid for diagnosis of deep-seated primary central nervous system lymphoma without histopathological confirmation. Acta Neurochir. (Wien) 157(2), 187-194 (2015).

15. Rosenfeld SS, Hoffman JM, Coleman RE, Glantz MJ, Hanson MW, Schold SC. Studies of primary central nervous system lymphoma with fluorine-18-fluorodeoxyglucose positron emission tomography. J. Nucl. Med. 33(4), 532-536 (1992).

16. Karantanis D, O'Eill BP, Subramaniam RM et al. 18F-FDG PET/CT in primary central nervous system lymphoma in HIV-negative patients. Nucl. Med. Commun. 28(11), 834-841 (2007).

17. Kosaka N, Tsuchida T, Uematsu H, Kimura H, Okazawa H, Itoh H. 18F-FDG PET of common enhancing malignant brain tumors. AJR Am. J. Roentgenol. 190(6), 365-369 (2008).

18. Kawai N, Zhen HN, Miyake K, Yamamaoto Y, Nishiyama Y, Tamiya T. Prognostic value of pretreatment 18F-FDG PET in patients with primary central nervous system lymphoma: SUV-based assessment. J. Neurooncol. 100(2), 225-232 (2010).

19. Kawase Y, Yamamoto Y, Kameyama R, Kawai N, Kudomi N, Nishiyama Y. Comparison of 11C-methionine PET and 18F-FDG PET in patients with primary central nervous system lymphoma. Mol. Imaging Biol. 13(6), 1284-1289 (2011).

20. Makino K, Hirai T, Nakamura $\mathrm{H}$ et al. Does adding FDG-PET to MRI improve the differentiation between primary cerebral lymphoma and glioblastoma? Observer performance study. Ann. Nucl. Med. 25(6), 432-438 (2011).

21. Das K, Mittal BR, Vasistha RK, Singh P, Mathuriya SN. Role of (18)F-fluorodeoxyglucose positron emission tomography scan in differentiating enhancing brain tumors. Indian J. Nucl. Med. 26(4), 171-176 (2011).

22. Ghesquieres H, Ferlay C, Richioud B et al. Usefulness of positron emission tomography using 18F-FDG performed at diagnosis and during initial chemotherapy for imaging primary central nervous system lymphoma: a 24 immunocompetent patient's study. Presented at: 53rd ASH Annual Meeting and Exposition. CA, USA, 10-13 December 2011.

23. Okada Y, Nihashi T, Fujii M et al. Differentiation of newly diagnosed glioblastoma multiforme and intracranial diffuse large B-cell lymphoma using 11C Methionine and 18F-FDG PET. Clin. Nucl. Med. 37(9), 843-849 (2012).

24. Yamaguchi S, Hirata K, Kobayashi $\mathrm{H}$ et al. The diagnostic role of $18 \mathrm{~F}-\mathrm{FDG}$ PET for primary central nervous system lymphoma. Ann. Nucl. Med. 28(7), 603-609 (2014).

25. Mercadal S, Cortes-Romera M, Velez P, Climent F, Gamez C, Gonzalez-Barca E. Positron emission tomography combined with computed tomography in the initial evaluation and response assessment in primary central nervous system lymphoma. Med. Clin. (Barc.) 144(11), 503-506 (2015). 
26. Wang $\mathrm{K}$, Zhao X, Chen Q et al. A new diagnostic marker for differentiating multicentric gliomas from multiple intracranial diffuse large B-cell lymphomas on 18F-FDG PET images. Medicine (Baltimore) 96(32), e7756 (2017).

27. Purandare NC, Puranik A, Shah S et al. Common malignant brain tumors: can 18F-FDG PET/CT aid in differentiation? Nucl. Med. Commun. 38(12), 1109-1116 (2017).

28. de-Bonilla-Damia A, Fernandez-Lopez R, Capote-Huelva FJ, de la Cruz-Vicente F, Egea-Guerrero JJ, Borrego-Dorado I. Role of 18F-FDG PET/CT in primary brain lymphoma. Rev. Esp. Med. Nucl. Imagen. Mol. 36(5), 298-303 (2017).

29. Okuyucu K, Alagoz E. Utility of FDG-PET/CT in primary central nervous system lymphoma and its contribution to prognosis estimation. Gulhane Med. J. 59(2), 44-48 (2017).

30. Albano D, Bosio G, Bertoli M, Giubbini R, Bertagna F. 18F-FDG PET/CT in primary brain lymphoma. J. Neurooncol. 136(3), 577-583 (2018).

31. Zhou W, Wen J, Hua F et al. 18F-FDG PET/CT in immunocompetent patients with primary central nervous system lymphoma: differentiation from glioblastoma and correlation with DWI. Eur. J. Radiol. 104, 26-32 (2018).

32. Zou Y, Tong J, Leng H, Jiang J, Pan M, Chen Z. Diagnostic value of using (18)F-FDG PET and PET/CT in immunocompetent patients with primary central nervous system lymphoma: a systematic review and meta-analysis. Oncotarget 8(25), 41518-41528 (2017).

-• Systematically summarizes and synthesizes the existing body of evidence assessing the diagnostic value of flouro-deoxy-glucose positron emission tomography/computed tomography in patients with PCNSL.

33. Baraniskin A, Schroers R. Modern cerebrospinal fluid analyses for the diagnosis of diffuse large B-cell lymphoma of the CNS. CNS Oncol. 3(1), 77-85 (2014).

34. Malani R, Bhatia A, Wolfe J, Grommes C. Staging identifies non-CNS malignancies in a large cohort with newly diagnosed lymphomatous brain lesions. Leuk. Lymphoma 60(9), 2278-2282 (2019).

- A large retrospective single-institution study that reports on the moderate yield of extensive systematic staging in patients with PCNSL in terms of identifying non-CNS malignancies and its implications.

35. Suh SC, Kim HS, Park JE, Jung SC, Choi CG, Kim SJ. Primary central nervous system lymphoma: diagnostic yield of whole-body CT and FDG-PET/CT for initial systemic staging. Radiology 292(2), 440-446 (2019).

- A single-institutional retrospective study that reports very low diagnostic yield of systemic staging in PCNSL and a higher rate of false positive findings resulting in further unnecessary procedures re-igniting the debate on the extent of systemic staging required in patients with PCNSL.

36. Yamashita K, Yoshiura T, Hiwatashi A et al. Differentiating primary CNS lymphoma from glioblastoma multiforme: assessment using arterial spin labelling, diffusion-weighted imaging, and 18F-flourodeoxyglucose positron emission tomography. Neuroradiology 55(2), 135-143 (2013).

37. Lin X, Lee M, Buck O et al. Diagnostic accuracy of T1-weighted dynamic contrast-enhanced-MRI and DWI-ADC for differentiation of glioblastoma and primary CNS lymphoma. AJNR Am. J. Neuroradiol. 38(3), 485-491 (2017).

38. Xu W, Wang Q, Shao A, Xu B, Zhang J. The performance of MR perfusion-weighted imaging for the differentiation of high-grade glioma from primary central nervous system lymphoma: a systematic review and meta-analysis. PLoS ONE 12(3), e0173430 (2017).

39. Alcaide-Leon P, Dufort P, Geraldo AF et al. Differentiation of enhancing glioma and primary central nervous system lymphoma by texture-based machine learning. AJNR Am. J. Neuroradiol. 38(6), 1145-1150 (2017).

40. Adams MC, Turkington TG, Wilson JM, Wong TZ. A systematic review of factors affecting accuracy of SUV measurements. AJR Am. J. Roentegol. 195(2), 310-320 (2010). 